\title{
USO DE PLANTAS MEDICINAIS POR IDOSOS PORTADORES DE HIPERTENSÃO ARTERIAL
}

\author{
Andréa Raquel Fernandes Carlos da Costa ${ }^{\text {* }}$ \\ Francidalva Moraes Cordovil \\ Márcia Jaqueline de Lima III \\ Wesley Adson Costa Coelho IV \\ Eucláudio Cavalcanti Salvador Filho ${ }^{\mathrm{V}}$
}

\begin{abstract}
RESUMO
É comum a população idosa buscar plantas com propriedades diuréticas e calmantes para prevenir ou controlar diversas doenças, entre estas a elevação da pressão arterial. Assim, para que se possa evitar riscos quanto ao uso de plantas medicinais pela população idosa que sofre de hipertensão arterial propôs-se estudar a utilização destas plantas, suas indicações terapêuticas e os riscos associados ao uso destas. A pesquisa foi um estudo quantitativo, de caráter descritivo e exploratório e de corte transversal e foi realizada em Centro Geriátrico e Centro de Referência de Assistência Social, localizados no município de Mossoró-RN. O estudo foi enviado para comitê de ética e após aprovação realizada coleta de dados. O levantamento dos dados foi realizado entre os meses de setembro a novembro de 2017, por meio de aplicação de formulário contínuo, sendo a pesquisa constituída de 37 idosos. Os dados foram expressos em valores de média, bem como frequência simples e percentagem. A presente pesquisa levantou informações relevantes sobre os riscos associados ao uso das plantas medicinais por idosos portadores de hipertensão arterial, e permitiu a orientação quanto ao uso correto. Os idosos fazem uso de plantas medicinais, com a finalidade de prevenir ou tratar alguma doença. Mesmo possuindo o conhecimento empírico sobre o uso destas, consomem pela automedicação, acreditando no poder da prevenção, tratamento e cura de doenças. Os resultados obtidos neste estudo reforçam a importância de programas de educação em saúde direcionados aos idosos, por ser esta faixa etária vulnerável.
\end{abstract}

PALAVRAS-CHAVE: Saúde do Idoso. Plantas Medicinais. Hipertensão Arterial. Envelhecimento.

Engenheira Agrônoma, Professora Doutora da Faculdade Nova Esperança de Mossoró - FACENE/RN. I Mossoró, RN, Brasil. ORCID: 0000-0001-9128-5926 Autor correspondente: andreacosta@facenemossoro.com.br Enfermeira, formada pela Faculdade Nova Esperança de Mossoró - FACENE/RN. Mossoró, RN, Brasil. II ORCID: 0000-0002-8680-3861

Enfermeira. Professora da Faculdade Nova Esperança de Mossoró - FACENE/RN. Mossoró, RN, Brasil. III ORCID: 0000-0002-4924-0502 Acadêmico do Curso de Enfermagem da Faculdade Nova Esperança de Mossoró - FACENE/RN, Brasil. IV ORCID: 0000-0003-1069-9321

Médico veterinário. Professor Doutor da Faculdade Nova Esperança de Mossoró - FACENE/RN, Brasil. ${ }^{V}$ ORCID: 0000-0001-9130-4488 


\section{INTRODUÇÃO}

Desde a Antiguidade, o homem faz uso de plantas medicinais com a finalidade de prevenir e curar diversas doenças. Essa tradição iniciou-se na China há mais de 3000 anos a.C, prática essa que até hoje é utilizada pelo conhecimento herdado de seus antepassados ${ }^{1}$.

No Brasil, a utilização popular das plantas medicinais é originada dos povos indígenas, que as utilizavam tanto para fins terapêuticos, quanto para rituais religiosos. Os africanos, junto com os índios e europeus, foram os responsáveis pela formação da base do conhecimento cultural e biológico acerca das plantas úteis no Brasil2.

Esse conhecimento foi sendo repassado de geração para geração entre os povos, nações e tribos. Todavia, com o decorrer dos anos e o advento da medicina, este conhecimento passou por algum tempo desvalorizado pelos profissionais de saúde que começaram a enfocar no tratamento alopático 3 .

Entretanto, o alto custo destes fármacos, o difícil acesso, os seus efeitos colaterais, bem como nos dias atuais, o uso crescente de produtos de origem natural, contribuíram para o ressurgimento do uso de plantas medicinais 4.

A utilização destas plantas com propriedades terapêuticas ocorre com maior frequência em famílias ou comunidades de baixa renda, que acabam encontrando alternativas em tratamentos naturais, sem ao menos conhecer os efeitos colaterais e os riscos que esses medicamentos possam oferecer. São pessoas com poucas informações quanto a sua forma de utilização e seus princípios tóxicos presentes 5 .

Em grande parte, essas pessoas são grupo de idosos, o que certamente desperta inúmeras preocupações, pois os idosos estão em processo de degeneração orgânica, o que de certa forma dificulta o curso dos princípios ativos das ervas no organismo, além de muitos possuírem órgãos cujo funcionamento já não é suficientemente adequado 5 .

São comumente usadas por essa população plantas com propriedades diuréticas e calmantes para prevenir ou controlar a elevação da pressão arterial $^{6}$. Em parte, isso se deve ao fato das dificuldades de adesão ao tratamento convencional (farmacológico), contribuindo para as taxas de abandono e busca de outros métodos.

Porém, o consumo de determinada droga vegetal, pode ser um risco para o idoso devido as possíveis reações adversas provocadas. Algumas das consequências negativas do uso de medicações, sem critérios, sejam fitoterápicos ou medicamentos alopáticos são a desidratação, toxidade pelo uso constante, gastrite, hipotensão e sedação, que devem ser constantemente avaliadas?.

Outro aspecto que deve ser ressaltado é que a planta somente apresenta valor medicinal, quando usada de maneira correta, devido aos riscos de intoxicação e surgimento de efeitos colaterais, já que estas produzem substâncias químicas que podem atuar beneficamente ou agirem de forma tóxica sobre o organismo. Assim, deve-se atentar quanto ao seu uso diário, dosagem e intervalos, evitando maiores complicações7.

Nesse sentido, para que se possa evitar riscos, quanto ao uso de plantas medicinais pela população idosa com hipertensão arterial, propôs-se estudar a utilização destas plantas entre idosos e os seus efeitos aliando o conhecimento popular ao científico. Apesar de boa parte da população fazer o uso de plantas medicinais, ainda são poucos os estudos que abordam a questão do uso dos recursos da flora por idosos, sendo necessário um maior investimento por parte dos pesquisadores na busca de fornecer informações as pessoas. 


\section{MATERIAL E MÉTODOS}

A pesquisa trata-se de um estudo quantitativo, de caráter descritivo e exploratório, de corte transversal e foi realizada no Centro Geriátrico Dia Madalena Aires e no Centro de Referência de Assistência Social (CRAS/ Alto do São Manoel), localizados no município de Mossoró-RN, após a aprovação do comitê de ética. O Centro Geriátrico e o CRAS são de responsabilidade da Secretaria Municipal de Desenvolvimento Social de Mossoró/RN.

A escolha dos locais deu-se pelo fato de apresentarem um maior número de atendimentos a idosos e, desta forma, as avaliações permitirão abranger um maior número de pessoas na terceira idade. A informação sobre o número de usuários idosos cadastrados em cada unidade foi obtida por meio da Secretária Municipal de Desenvolvimento Social de Mossoró/RN.

A população de estudo foi constituída por idosos do município de Mossoró/RN. Para coleta de dados, os idosos foram escolhidos aleatoriamente, obedecendo ao critério da abordagem oportuna.

Considerando-se uma população de 37 elementos, a amostra foi não probabilística por conveniência, na qual trabalhou-se com a totalidade ( $n=37)$ adotando-se, dentre a população selecionada, um total de 37 idosos.

A partir da adesão espontânea ao convite para participação na pesquisa, foi realizada a aplicação do formulário, possibilitando reunir informações que interessam ao estudo. Os critérios de inclusão utilizados no estudo foram homens e mulheres que estavam:

- $\quad$ Cadastrados no local de estudo;

- $\quad$ Idade igual ou superior a 60 anos;

Já os critérios de exclusão foram:
- Doença física ou mental que impossibilitassem a aplicação do formulário, bem como a ausência dos idosos nos dias de coleta de dados

O levantamento de dados foi realizado nos meses de setembro a novembro de 2017, por meio de aplicação de formulário contínuo, elaborado com base em autores como Oliveira et al. ${ }^{8}$ e Silva-Hah 9 . O formulário foi respondido pelos idosos cadastrados no Centro Geriátrico Dia Madalena Aires e no Centro de Referência de Assistência Social (CRAS/Alto do São Manoel) no município de Mossoró/RN, com prévia autorização escrita. Os idosos foram informados sobre o anonimato na utilização dos dados para a pesquisa e que, qualquer dúvida sobre o presente estudo, poderia ser esclarecida com a pesquisadora associada e pesquisadora responsável. O formulário constou de questões abrangentes (perfil socioeconômico e demográfico) e questões específicas sobre o consumo de plantas pelos idosos.

Os dados foram expressos em valores de média, bem como frequência simples e percentagem por meio do programa Microsoft Excel, verificando a utilização de plantas medicinais pelos idosos, os tipos de plantas mais consumidas, acompanhamento e indicação do médico e entre outras variáveis.

Quanto aos aspectos éticos, o estudo seguiu os termos da Resolução do Conselho Nacional de Saúde (RNS) 466/2012, que trata das diretrizes e normas regulamentadoras de pesquisas que envolvem seres humanos com interesse organizado, de caráter consultivo, educativo e formulador de diretrizes e estratégias no âmbito do conselho e foi submetido ao Comitê de Ética e Pesquisa (CEP) da Faculdade de Enfermagem e Medicina Nova Esperança/ FACENE/PB, e aprovado sob número de protocolo CAAE: 78985317.9.0000.5179 e núme- 
ro de parecer 2.460.303. Após aprovação do projeto pelo Comitê de Ética e Pesquisa (CEP) da Faculdade de Enfermagem Nova Esperança

\section{RESULTADOS E DICUSSÃO}

A média de idade dos idosos do estudo foi de $46 \%$ entre 70 a 79 anos, sendo que $24 \%$ eram do sexo masculino e $76 \%$ feminino (TABELA 1). Resultado semelhante foi encontrado por Feijó et.al. ${ }^{3}$, ao avaliar a utilização de plantas medicinais utilizadas por idosos com diagnóstico de Diabetes Mellitus no tratamento dos sintomas da doença, no qual identificaram maiores percentuais de idosos do sexo feminino (55\%) com idade entre 60 e 77 anos. Tais resultados podem ser explicados pelo fato de uma maior participação das mulheres no grupo de idosos, levando a crer que as idosas aparentam um maior interesse em participar de atividades educativas e de lazer, ou ainda que, mesmo após a aposentadoria, os homens procuram exercer alguma profissão, preferindo dedicar seu tempo livre a alguma atividade remunerada, sobrando menos tempo para se dedicar a atividades de lazer e aos recursos da comunidade ${ }^{6}$.

A maioria dos idosos são viúvos (43\%) e apresentam renda familiar de até 1 salário mínimo (73\%). Alguns autores, ao avaliar a utilização de plantas medicinais, com atividade antimicrobiana por usuários do serviço público de saúde em Campina Grande - Paraíba, identificaram que a renda familiar $61,4 \%(n=135)$ dos pesquisados situou-se entre 1 e 2 salários mínimos10. Tal fato, provavelmente seja explicado pelo baixo poder aquisitivo da população, que não tem como custear suas necessidades básicas de saúde.

Em relação ao grau de escolaridade, a maior parte dos idosos possui ensino básico
VOLUME 17 - NÚMERO 1 - ABR/2019 ISSN ELETRÔNICO 2317-7160 19
(41\%). Foi verificado que era maior o número de mulheres idosas com ensino básico (67\%), em relação aos homens idosos (33\%). O maior uso de plantas medicinais entre as mulheres, evidencia que estas são maiores detentoras de conhecimento sobre o uso de ervas com propriedades medicinais. Re tratando com este um fato histórico, já que, o uso de ervas medicinais remonta a tribos primitivas em que as mulheres estavam à frente de extrair os princípios ativos de plantas para usá-los na cura de doenças ${ }^{11}$.

Em se tratando da prática de atividade física foi verificado neste artigo que $73 \%$ dos idosos praticam exercícios físicos e $27 \%$ não praticam, isto pode ser explicado pelo fato de que um programa de exercícios nesta faixa etária tem o objetivo de elevar a qualidade de vida destes indivíduos, além de preservar ou melhorar a autonomia, bem como minimizar ou retardar os efeitos da idade, mostrando a importância de respeitar sempre as limitações próprias desta fase da vida, para que se possa obter os resultados e benefícios esperados, tanto físicos quanto psicológicos ${ }^{12}$.

Com relação ao uso de tabaco ou bebida alcoólica, $92 \%$ da população idosa não faz uso, apresentando benefícios à saúde destes. Corroborando com este estudo poucas são as pesquisas que relatam o impacto do uso de álcool e cigarro na vida dos idosos, especialmente nos países em desenvolvimento, como o Brasil, em que a população idosa vem crescendo ${ }^{13}$.

Porém, do ponto de vista da Saúde Pública, os cinco mais importantes fatores de risco para doenças crônicas não-transmissíveis são o 
tabagismo, o consumo de álcool, a obesidade ou sobrepeso, sedentarismo e a dislipidemia ${ }^{13}$.

O tabaco é um dos mais potentes agentes carcinogênicos para o ser humano e seu consumo, assim como a exposição a fumaça produzida pelo fumante, são identificados como a maior causa passível de prevenção de doenças. O consumo aumentado de álcool está associado à hipertensão arterial, à cirrose, ao acidente vascular hemorrágico e aos cânceres da orofaringe, laringe, esôfago e fígado ${ }^{13}$.

TABELA 1- Caracterização dos idosos do Centro Geriátrico Dia Madalena Aires e do Centro de Referência de Assistência Social Alto do São Manoel - Mossoró/RN.

\begin{tabular}{|c|c|c|}
\hline Variáveis & n (pessoas) & $\%$ \\
\hline \multicolumn{3}{|l|}{ Gênero } \\
\hline Homem & 9 & 24,0 \\
\hline Mulher & 28 & 76,0 \\
\hline \multicolumn{3}{|l|}{ Idade } \\
\hline $60-69$ & 14 & 1 \\
\hline $70-79$ & 17 & 4 \\
\hline $80-89$ & 5 & 13,0 \\
\hline $90-99$ & 1 & 3,0 \\
\hline \multicolumn{3}{|l|}{ Estado Civil } \\
\hline Casado(a) & 9 & 24,0 \\
\hline Solteiro(a) & 7 & 19,0 \\
\hline Separado(a)/divorciado) & 5 & 14,0 \\
\hline União estável & 0 & 0,0 \\
\hline Viúvo & 16 & 43,0 \\
\hline \multicolumn{3}{|l|}{ Renda Familiar } \\
\hline Até 1 salário mínimo & 27 & 73,0 \\
\hline De 1 a 3 salários mínimos & 10 & 27,0 \\
\hline 3 a 5 salários mínimos & 0 & 0,0 \\
\hline Mais de 5 salários mínimos & 0 & 0,0 \\
\hline \multicolumn{3}{|l|}{ Grau de escolaridade } \\
\hline Sem estudo & 4 & 11,0 \\
\hline Ensino básico & 15 & 41,0 \\
\hline Ensino fundamental & 10 & 27,0 \\
\hline Ensino médio & 6 & 16,0 \\
\hline Ensino superior & 2 & 5 \\
\hline
\end{tabular}




\section{Pratica alguma atividade física?}

Sim

Não

Faz uso de tabaco ou bebida alcoólica?

Sim

Não
73,0

27,0

8,0

92,0
Ao avaliar o número de idosos com hipertensão, identificou-se que $43 \%$ da população possui hipertensão arterial (TABELA 2). Número bastante significativo, visto que na grande maioria das vezes é assintomática e quando existem sintomas, geralmente apresentam lesões de órgãos-alvo ${ }^{14}$.

O elevado número de pacientes com idade superior a 60 anos é característica de populações portadoras de hipertensão, fato corroborado pelas VII Diretrizes Brasileiras de Hipertensão15, que sugerem relação entre elevação dos níveis pressóricos e a idade, com prevalência de Hipertensão Arterial Sistêmica superior a $60 \%$ na faixa etária acima de 65 $\operatorname{anos}^{16}$.

No que diz respeito ao resultado ob- tido, em relação ao uso de medicamentos alopáticos, $43 \%$ dos idosos fazem uso destes, sendo utilizado com mais frequência Lozartana Potássica (22\%) e geralmente grande parte dos idosos faz o uso de anti-hipertensivos entre uma a duas vezes ao dia (44\%).

Os idosos hipertensos devem ser tratados objetivando a redução da pressão arterial (PA). A introdução de anti-hipertensivo deve ser feita por médicos em doses baixas e, se necessário, com aumento gradual, porém sem perder de vista o alvo de PA desejado. Os pacientes devem ser educados em relação a doença, durante as consultas médicas, sempre que possível em grupos com assistência multiprofissional $^{16}$.

TABELA 2- Caracterização dos idosos com relação a Hipertensão Arterial do Centro Geriátrico Dia Madalena Aires e do Centro de Referência de Assistência Social Alto do São Manoel - Mossoró/RN.

\begin{tabular}{lc|c}
\hline \hline \multicolumn{1}{c|}{ Variáveis } & n (pessoas) & $\%$ \\
\hline \hline Idosos com hipertensão arterial & & \\
Sim & 16 & 43,0 \\
Não & 21 & 57,0 \\
\multicolumn{2}{l}{ Medicamento alopático da Hipertensão Arterial } & \\
Sim & 16 & 43,0 \\
Não & 21 & 57,0 \\
Tipos de medicamentos alopático para Hipertensão arterial & \\
Enalapril & 3 & 19,0 \\
Captopril & 3 & 19,0 \\
Atenalol & 2 & 12,0
\end{tabular}




\section{Tipos de medicamentos alopático para Hipertensão arterial}

Candesartana cilexetila

Lozartana potássica

Succinato de metoprolol

Não lembra

\section{Quantas vezes ao dia}

$1 \times$ ao dia

$2 \times$ ao dia

$3 \times$ ao dia

Não lembra

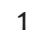

4

1

2

7

7

0

2
6,5

25,0

6,5

12,0

44,0

44,0

0,0

12,0
O Brasil tem uma rica história de uso das plantas medicinais no tratamento dos problemas de saúde da população, uso este construído com base na experiência popular, sendo transmitido através de gerações ${ }^{17}$.

As plantas medicinais correspondem as mais antigas "armas" empregadas pelo homem no tratamento de enfermidades de todos os tipos, ou seja, a utilização de plantas na prevenção e/ou na cura de doenças é um hábito que sempre existiu na história da humanidade ${ }^{18}$.

Quanto a utilização das plantas medicinais, foi observado que $81 \%$ dos idosos as utilizam com a finalidade de prevenir, tratar alguma enfermidade (FIGURA 1), sendo 53\% destes portadores de hipertensão arterial.

FIGURA 1- Uso de Plantas Medicinais por idosos do Centro Geriátrico Dia Madalena Aires e do Centro de Referência de Assistência Social Alto do São Manoel - Mossoró/RN.

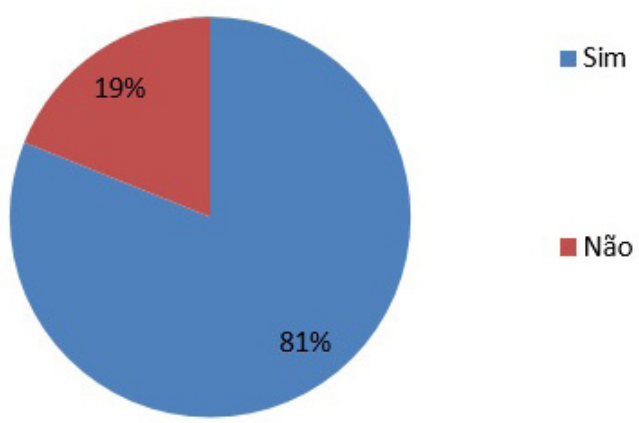

As plantas medicinais com maior prevalência no grupo de idosos foram: erva cidreira (17\%), boldo (13\%), hortelã (13\%), capim santo (13\%), camomila ( $8 \%$ ). Porém, não houve nenhuma associação com a hipertensão arterial diferindo assim da literatura, pois al- gumas destas plantas podem auxiliar no tratamento da hipertensão arterial, desde que tenha orientação de profissionais da saúde para fazer o uso de forma correta.

A Melissa Officinalis L. vem da Família Botânica chamada Lamiaceae (Labiatae), 
popularmente conhecida como melissa, erva-cidreira ou cidreira. As partes utilizadas desta planta são as folhas, ou a própria planta florida as quais são empregadas na forma de infusão, decocção, ou por extrato fluido. São de propriedade carminativa, estimulante, tônico, sedativo, anti-histérico. ${ }^{19}$ A Anvisa reitera que ela pode potencializar o efeito de medicamentos sedativos. Possui também atividade fungicida, bactericida, antidiarreica, atua como anti-inflamatório, antiespasmódico, hipotensor, anticonvulsivo, analgésico, antiemético, antirreumático, antisséptico e tratamento de desordens nervosas e febres e gastrointestinais ${ }^{20}$.

O Pneumus Boldus (boldo) é indicado para o tratamento de problemas no fígado, digestão, gastrite e azia, porém deve-se ficar bastante atento ao seu uso pois, segundo relato de alguns autores, o boldo eleva a pressão arterial, daí a importância do conhecimento das ervas e da indicação por profissional de saúde ${ }^{20}$. Possui também efeito antioxidante. Em sua composição, há uma concentração relativa de alcaloides e fenólicos de boldo e suas atividades sugerem que o efeito de eliminação de radicais livres é principalmente devido a catequina e flavonoides. Já o efeito antioxidante está relacionado principalmente com o conteúdo de catequinas ${ }^{21}$.

Mentha x Villosa L., conhecida como hortelã, é uma erva aromática que possui suas folhas na cor verde escura. Essa planta contém óleo essencial principalmente em suas folhas. Sua ação pode ser como antiespasmódico, anti-inflamatório e antiviral. Sendo também utilizada para tratar a má-digestão e sensação de flatulência, formação de gases. O chá é preparado principalmente por infusão e com as folhas frescas, para evitar que seus princípios ativos se evaporem. Utiliza-se 1,5 g de folhas em $150 \mathrm{ml}$ de água para o preparo do chá e recomenda-se utilizar de 2 a
4 vezes por $\mathrm{dia}^{22}$.

A hortelã é citada, pela maioria dos idosos, para melhorar a digestão e utilizada em casos de verminoses, resultado este coincidente com a literatura. Entre os diversos efeitos colaterais que podem ser desencadeados pelo uso indiscriminado da Hortelã está a inibição da absorção do ferro, potencialização da ação de fármacos, aumento dos hormônios folículo estimulante e testosterona, além de minimizar efeito antissupressor no organismo $^{18}$.

O Cymbopogon Citratus (DC) Stapf. é conhecido popularmente por diversos nomes como capim-limão, capim-santo, capim-cidreira e capim de cheiro, originário do veIho mundo, porém muito cultivado no Brasil. É uma erva aromática cujas folhas possuem um cheiro que lembra o limão. O chá preparado dessa planta tem ação calmante e uma ação analgésica. Pode ser usado em crises de cólicas uterinas e intestinais por conter uma substância conhecida como mirceno, como calmante suave $\mathrm{e}^{20}$. O chá do capim santo é feito por infusão, pois se utiliza as folhas. A RDC 10 de 2010 preconiza utilizar de 1 a $3 g$ da folha em $150 \mathrm{ml}$ de água e fazer a ingestão de uma xícara de chá 2 a 3 vezes ao dia23. o Cymbopogon Citratus (DC) Stapf tem se mostrado eficaz no auxílio do tratamento da hipertensão arterial, possui efeito calmante, antiespasmolitica, analgésico e bom sinergismo com antibióticos. Essas ações terapêuticas são produzidas, principalmente, pelo citral, composto do óleo essencial desta erva 24 .

Conhecida popularmente como camomila-vulgar, camomila, camomila-alemã, maçania ou maçanilia a Matricaria Chamomilla é uma planta da família Asteraceae. A camomila é originária da Europa. É indicada para má digestão, cólica uterina, sedativa, para queimaduras de sol, conjuntivite e olhos cansados (uso de compressas), vermífugo, dores musculares, tensão menstrual, estres- 
se, insônia, diarreia, inflamações das vias urinárias. Misturado ao chá de hortelã com mel, é utilizada no combate gripes e resfriados, para hemorroidas, para o fígado, antialérgico, dores de reumatismos, nevralgias e age como sudorífico. Estudos de M. Chamomilla em seres humanos são limitados e os estudos clínicos para examinar as supostas propriedades sedativas do chá de camomila estão ausentes 25 .
Principal modo de preparo das plantas medicinais foi a infusão com $70 \%$, utilizada uma vez ao dia (44\%), pois são naturais e não fazem mal a saúde ${ }^{24}$ (TABELA 3). Outro estudo mostra o modo de preparo: como primeira escolha foi relatada a infusão, essa técnica ocorre com a fervura da água, seguida da imersão da planta e abafamento por tempo determinado ${ }^{18}$.

TABELA 3- Caracterização dos idosos quanto a utilização das Plantas Medicinais do Centro Geriátrico Dia Madalena Aires e do Centro de Referência de Assistência Social Alto do São Manoel - Mossoró/RN.

\begin{tabular}{|c|c|c|}
\hline Variáveis & n (pessoas) & $\%$ \\
\hline \multicolumn{3}{|c|}{ Usa ou já fez uso de plantas medicinais (chás)? } \\
\hline $\operatorname{Sim}$ & 30 & 81,0 \\
\hline Não & 7 & 19,0 \\
\hline \multicolumn{3}{|l|}{ Como prepara? } \\
\hline Infusão & 21 & 70,0 \\
\hline Decocção & 8 & 27,0 \\
\hline Maceração & 1 & 3,0 \\
\hline Outros & 0 & 0,0 \\
\hline \multicolumn{3}{|c|}{ Qual motivo do uso das plantas medicinais? } \\
\hline Mal estar & 6 & 20,0 \\
\hline Doença & 6 & 20,0 \\
\hline $\begin{array}{l}\text { Complemento ao tratamento da } \\
\text { hipertensão arterial }\end{array}$ & 2 & 7,0 \\
\hline Opção & 0 & 0 \\
\hline Preferência & 13 & 43,0 \\
\hline Insônia & 3 & 10,0 \\
\hline \multicolumn{3}{|c|}{ Quantas vezes faz uso das plantas medicinais? } \\
\hline $1 \times$ ao dia & 13 & 44,0 \\
\hline $2 \times$ ao dia & 7 & 23,0 \\
\hline $3 \times$ ao dia & 1 & 3,0 \\
\hline $1 \mathrm{x}$ por semana & 2 & 7,0 \\
\hline $2 \times$ por semana & 1 & 3,0 \\
\hline $3 \times$ por semana & 4 & 14,0 \\
\hline $4 \times$ por semana & 1 & 3,0 \\
\hline
\end{tabular}


Efeito colateral após o uso de plantas medicinais?

Sim

O uso das plantas medicinais teve acompanhamento de algum profissional de saúde?

Você informa ao seu médico o hábito de tomar chás medicinais?

Foi prescrito por quem?

Parente/vizinho

$\begin{array}{cc}5 & 17,0 \\ 1 & 3,0 \\ 24 & 80,0 \\ 0 & 0,0 \\ 0 & 0,0\end{array}$

TV/internet

Tem conhecimento sobre os possíveis riscos procedentes do uso indevido ou incorreto de plantas medicinais? ?

Sim

Entretanto, os profissionais de saúde devem ficar atentos a este uso, já que são comuns os conceitos equivocados a respeito de sua segurança e finalidade. $O$ fato de uma substância ser designada "natural" não quer dizer que ela seja isenta de riscos ${ }^{26}$.

Quanto ao motivo de uso, $20 \%$ dos idosos utilizam para mal-estar $43 \%$ por preferência, $20 \%$ por doença, $7 \%$ por complemento ao tratamento da PA e 10\% por insônia. Com relação ao efeito colateral, $100 \%$ dos idosos não sentem nenhum efeito colateral após o uso, e ainda assim, afirmam que não informam ao médico sobre o hábito de tomar chás medicinais (67\%). Por outro lado, a falta de comunicação entre médico e paciente pode dificultar o monitoramento de possíveis reações adversas e interações medicamentosas, sendo necessário que os profissionais da área da saúde saibam questionar seus pacientes não só sobre os medicamentos convencionais, mas também sobre o uso de plantas medicinais 27 .

Além disso, $80 \%$ dos idosos fazem uso das plantas por automedicação e $73 \%$ desconhecem os possíveis riscos do uso indevido, ou incorreto das plantas medicinais. As plantas medicinais devem ser utilizadas com muito cuidado, pois não é apenas um "chazinho", elas têm muitos efeitos, inclusive a toxicidade quando utilizada por muito tempo a mesma planta. Não se pode comprar plantas medicinais em qualquer lugar ou utilizar a planta de qualquer lugar, pois tem os riscos de contaminação 27.

A identificação errônea das espécies vegetais, forma de preparo incorreta e o uso indiscriminado também são perigosos, po- 
dendo levar a superdosagem, ineficácia terapêutica e efeitos indesejáveis, o que pode acarretar sérios danos a saúde ${ }^{28}$.

Verificou-se que a utilização de plantas medicinais por idosos, ainda é bastante difundida, fazendo-se necessária a realização de estudos que busquem resgatar o saber popular relacionado com o conhecimento científico, visto que, seja levado em consideração no planejamento das políticas de saúde em nosso país, norteando estratégias que visem a boa execução dessa terapêutica complementar ${ }^{28}$. Além disso, é importante destacar que a fitoterapia continua sendo utilizada como instrumento terapêutico, dispondo de medicamentos fitoterápicos e drogas vegetais tão eficazes e seguras quanto os medicamentos sintéticos.

No entanto, o uso de plantas medicinais como coadjuvante para o tratamento de doenças crônicas requer estudos farmacológicos preliminares do quadro clínico de cada indivíduo, por profissional habilitado, bem como orientações acerca de seu uso racional e possíveis interações, uma vez que esse tipo de terapia também pode apresentar riscos associados $^{29}$.

\title{
CONCLUSÃO
}

Os idosos fazem uso de plantas medicinais com a finalidade de prevenir, ou tratar alguma doença. Mesmo possuindo o conhecimento empírico sobre o uso destas, estes consomem ervas medicinais pela automedicação, acreditando no poder da prevenção, tratamento e cura de doenças. Porém, a população idosa desconhece os efeitos tóxicos da utilização inadequada das plantas medicinais.

\section{USE OF MEDICINAL PLANTS BY ELDERLY WITH ARTERIAL HYPERTENSION}

\begin{abstract}
It is common for the elderly population to seek plants with diuretic and calming properties to prevent or control various diseases, including elevated blood pressure. Thus, in order to avoid risks related to the use of medicinal plants by the elderly population suffering from hypertension, it was proposed to study the use of these plants, their therapeutic indications and the risks associated with their use. The research was a quantitative, descriptive and exploratory cross-sectional study and was carried out at the Geriatric Center and the Social Assistance Referral Center located in the municipality of Mossoró-RN. The study was sent to the Ethics Committee and after approval was given the data collection started. Data collection was carried out between September and November 2017, through the application of a continuous form, and the survey consisted of 37 elderly people. The data were expressed in mean values as well as simple frequency and percentage. The present research raised relevant information on the risks associated with the use of these by elderly people with arterial hypertension, and allowed the orientation regarding the correct use of medicinal
\end{abstract}


plants. Therefore, it is evident that the elderly make use of medicinal plants, with the purpose of preventing or treating some disease. Even knowing about their use, they consume by self- medication, believing in the power of prevention, treatment and cure of diseases. It is concluded that the results obtained in this study reinforce the importance of health education programs directed to the elderly, because this is a vulnerable age group.

KEYWORDS: Elderly; Medicinal plants; Arterial hypertension; Aging.

\section{REFERÊNCIAS}

1. Braga CM. Histórico da Utilização de Plantas Medicinais [trabalho de conclusão de curso]. Brasília: Universidade de Brasília/Universidade Estadual de Goiás, 2011.

2. Silva NCB. Uso de plantas medicinais na comunidade quilombola da Barra II - Bahia, Brasil. Boletim Latinoamericano y del Caribe de Plantas Medicinales y Aromáticas. 2012;11(5):435-453.

3. Feijó $A M$, Bueno MEN, Ceolin T, Linck CL, Schwartz E, Lange C, Meincke SMK, Heck RM, Barbieir, RL, Heiden G. Plantas medicinais utilizadas por idosos com diagnóstico de Diabetes mellitus no tratamento dos sintomas da doença. Rev. Bras. Plantas Med. [online], 2008;14(1):5056.

4. Balbinot S. Reconhecimento e uso de plantas medicinais pelos idosos do Município de Marmeleiro - Paraná. Rev Bras Plantas Med. 2013;15(4):632-638.

5. Oliveira Junior RG. Plantas Medicinais Utilizadas Por Um Grupo De Idosos Do Município De Petrolina, Pernambuco. Revista Eletr. Farm. 2012;9(3):16 - 28.

6. Oliveira CJ. Plantas medicinais: usos e crenças de idosos portadores de hipertensão arterial. Rev. Eletr. Enf. 2007;9(1):93-105.
7. Ventura MF. Uso de Plantas Medicinais por Grupo de Idosos de Unidade de Saúde de Campo Grande Rio de Janeiro: uma discussão para a implementação da fitoterapia local: [trabalho de conclusão de curso]. Rio de Janeiro: FIOCRUZ; 2012.

8. Oliveira EB. Uso de Plantas Medicinais por Portadores de Hipertensão Arterial Sistêmica. Ensaios Cienc., Cienc. Biol. Agrar. Saúde. 2014;18(3):137-142.

9. Silva BQ. Uso de plantas medicinais por indivíduos com hipertensão arterial sistêmica, diabetes mellitus ou dislipidemias. R. Bras. Farm. Hosp. Serv. Saúde. 2011;2(3):36-40.

10. Souza CMP. Utilização de Plantas Medicinais com Atividade Antimicrobiana por Usuários do Serviço Público de Saúde em Campina Grande - Paraíba. Rev. Bras. Pl. Med. 2013;15(2):188-193.

11. Lima DF. Conhecimento e uso de plantas medicinais por usuários de duas unidades básicas de saúde. Rev Rene. 2014;15(3):383-390.

12. Argento RSV. Benefícios da atividade física na saúde e qualidade de vida do idoso [Trabalho de conclusão de curso]. Campinas, SP: [s.n], 2010.

13. Senger AEV. Alcoolismo e tabagismo em ido- 
sos: relação com ingestão alimentar e aspectos socioeconômicos. Rev. bras. geriatr. gerontol. 2011;14(4):713-719.

14. Jobim EFC. Hipertensão Arterial no Idoso: Classificação e peculiaridades. Rev. Bras. Clin. Med. 2008;6:250-253.

15. Sociedade Brasileira de Cardiologia. VII Diretrizes brasileiras de hipertensão. Arq Bras Cardiol. 2016;107(3 supl 3):1-51.

16. Nunes MGS. Uso de plantas medicinais por pessoas com hipertensão. Rev Rene; 2015;16(6):775-81.

17. Perrottil TC. Tratamento farmacológico da hipertensão no idoso. Rev. Bras Hipertens. 2001;14(1):37-41.

18. Firmo WC, Menezes VJ, Passos CEC, Dias CN, Alves LPL, Dias, ICL, Santos Neto M, Olea RSG . Contexto Histórico, Uso Popular e Concepção Científica Sobre Plantas Medicinais. Cad. Pesq. 2011.

19. Grando, TM. Tratado das plantas medicinais \{recurso eletrônico\}: mineiras, nativas e cultivadas. 1. ed. Dados eletrônicos. Belo Horizonte: Adaequatio Estúdio, 2014. Disponível em: https://plantasmedicinaismineiras.wordpress. com/. Acesso em março de 2019.

20. Szerwieski LLD. Uso de plantas medicinais por idosos da atenção primária. Rev. Eletr. Enf. [Internet]. 2017.

21. Schmeda-Hirschmann G, Rodriguez JA, Theoduloz C, Astudillo SL, Feresin GE, Tapia A. Free-radical scavengers and antioxidants from Peumus boldus Mol. ("Boldo"). Free Radic Res. 2003; 37(4):447-52.
22. Lorenzi H. Plantas Medicinais no Brasil: nativas e exóticas. $2^{\text {a }}$ ed. Nova Odessa: Instituto Plantarum. 2008.

23. Agência Nacional de Vigilância Sanitária (ANVISA). Dispõe sobre alimentos para atletas. Resolução n. 18, de 27 de março de 2010.

24. Pereira PS, Ruyz, LL, Paula J. Ações terapêuticas do capim-santo: uma revisão de literatura therapeutic actions of grass-saint: a literature review. Revista Saúde em Foco. 2018.

25. Evangelista CA. O Uso de Plantas Medicinais por Idosos Atendidos em Unidades de Saúde da Família da Região Sul e Palmas-TO [trabalho de conclusão de curso]. Palmas: Centro Universitário Luterano de Palmas, 2016.

26. Bett MS. O uso popular de plantas medicinais utilizadas no tratamento da ansiedade no município de Galvão-SC: [trabalho de conclusão de curso]. Florianópolis: Universidade Federal de Santa Catarina, 2013.

27. Teixeira K. Plantas medicinais que podem causar alteração na pressão arterial e interação com anti-hipertensivos [trabalho de conclusão de curso]. Criciúma: Universidade do Extremo Sul Catarinense; 2011.

28. Oliveira CJ. Caracterização do tratamento não-farmacológico de idosos portadores de hipertensão arterial. Rev. Rene. 2010;11(1):76-85.

29. Machado HL. Pesquisa e atividades de extensão em fitoterapia desenvolvidas pela Rede Fito Cerrado: uso racional de plantas medicinais e fitoterápicos por idosos em Uberlândia-MG. Rev. Bras. Plantas Med. 2014;16(3):527-533. 